\title{
RINGS OF MEROMORPHIC FUNCTIONS ${ }^{1}$
}

\author{
H. L. ROYDEN
}

1. Introduction. In this paper we discuss some of the algebraic properties of rings of meromorphic functions on Riemann surfaces of finite genus. We consider primarily the compact surfaces and those open surfaces which are open subsets of compact surfaces and have the property that each boundary point (relative to the compact surface) can be an essential singularity for a bounded analytic function on the open surface.

On the compact surfaces we are interested in the field of all meromorphic functions while on the open surfaces we are most interested in the field of quotients of the bounded analytic functions. For the sake of convenience we shall refer to both of these fields as the fields of "meromorphic functions of bounded characteristic."

In $\$ 3$ we characterize the valuations on this field for finite Riemann surfaces, i.e. connected open subsets of compact surfaces whose boundary consists of a finite number of Jordan curves. In the last section we characterize the homomorphisms of this field into the field of all meromorphic functions on an arbitrary Riemann surface.

Some of the present results have been anticipated by Heins [1], particularly Proposition 3 and the corollary to Proposition 4. The methods of proof used here are somewhat different and are closer to the work of Rudin [3].

2. Some function-theoretic lemmas. We list here some lemmas concerning Riemann surfaces. The first is classical [4], and the proof the third is quite similar. The second lemma is a consequence of Abel's theorem.

Lemma 1. Let $W$ be a compact Riemann surface and $f$ a meromorphic function on it. Then $f$ assumes each value on the Riemann sphere the same number of times, say $n$. If $g$ is any other meromorphic function on $W$, then $g$ satisfies an algebraic equation of degree $n$ whose coefficients are rational functions of $f$. Moreover, for each complex number $z$ we may choose a meromorphic function $g$ which separates the points $f^{-1}(z)$ and such that every meromorphic function on $W$ is a rational function of $f$ and $g$.

Received by the editors January 15, 1958, and, in revised form, March 21, 1958.

1 This work was sponsored by the Office of Ordnance Research, U. S. Army, and was presented to the Princeton Conference on Analytic Functions on September 9, 1957. 
Lemma 2. Let $W$ be a noncompact finite Riemann surface. Then for some integer $n$ there is an analytic function $f$ which maps $\bar{W}$ onto the circle $|z| \leqq 1$ so that each value of $z$ in $|z|<1$ is assumed exactly $n$ times.

Lemma 3. Let $W$ be a noncompact finite Riemann surface and $f$ the function of Lemma 2. Then each analytic function $g$ on $W$ satisfies an equation

$$
\sum_{\nu=0}^{n} b_{\nu}(f) g^{\nu}=0
$$

where the $b_{\nu}$ are analytic functions in the interior of the unit circle which are bounded if $g$ is bounded. For each $z$ in $|z|<1$ we can find a bounded analytic function $g$ on $W$ which separates the points $f^{-1}(z)$ and which has the property that each analytic function $h$ on $W$ can be expressed as

$$
h=\sum_{\nu=0}^{n-1} c_{\nu}(f) g^{\nu}
$$

where the coefficients $c_{\nu}$ are analytic functions in the interior of the unit circle and are bounded if $h$ is.

3. The valuations on some fields of meromorphic functions. Let $F$ be a field which contains the field $C$ of complex numbers. By a valuation on $F$ we shall mean a mapping $v$ of the nonzero elements of $F$ onto the integers such that

(i) $v(f g)=v(f)+v(g)$,

(ii) $v(f+g) \geqq \min [v(f), v(g)]$,

(iii) If $v(f) \geqq 0$, there is a $\lambda \in C$ such that $v(f-\lambda)>0$.

It should be noted that these properties imply that $v(\lambda)=0$ for each nonzero $\lambda$ in $C$. This implies that the $\lambda$ in (iii) is unique.

In this section we prove two propositions about valuations. The first is classical, but the proof is included here both for completeness and for comparison with the proof of the second proposition.

Proposition 1. Let $F$ be the field of meromorphic functions on a compact Riemann surface $W$. Then for each valuation $v$ on $F$ there is a unique point $p_{0} \in W$ such that $v(f)$ is the order ${ }^{2}$ of $f$ at $p_{0}$.

Proof. Let $f$ be a function in $F$ with $v(f)=1$, and let $p_{0}, p_{1}, \cdots, p_{k}$ be the zeros of $f$. For any rational function $r$, we have $v[r(f)]$ equal to the order of $r$ at zero. Let $g$ be any function in $F$. Then $g$ satisfies an equation

${ }^{2}$ By the order of a function we mean the order of vanishing or minus the order of a pole. 


$$
g^{n}+\sum_{\nu=0}^{n-1} r_{v}(f) g^{\nu}=0,
$$

where the $r_{\nu}$ are rational functions of $f$. If $v(g)$ were negative, then for some $\nu$ we have $v\left(r_{\nu}(f)\right)$ negative, whence $r_{\nu}$ has a pole at zero. Since $r_{\nu}(0)$ is an elementary symmetric function of the values of $g$ at the points $p_{i}$, we see that $g$ must have a pole at one of the points $p_{i}$. Consequently, if $g$ is neither zero nor infinite at any of the points $p_{i}$, we have $v(g)=0$.

Let $g$ be a function which separates the points $p_{i}$ and whose derivative does not vanish at any of the $p_{i}$. Set $g_{i}=g-g\left(p_{i}\right)$. If $\nu_{i}$ is the order of $f$ at $p_{i}$, the function $f /\left[\Pi g_{i}^{\nu_{i}}\right]$ is neither zero nor infinite at any of the $p_{i}$ must so must have zero valuation. Since $v\left(g_{i}\right) \geqq 0$, and

$$
1=v(f)=\sum_{i=0}^{k} \nu_{i} v\left(g_{i}\right),
$$

we have $v\left(g_{i}\right)=0$ for all $i$ except one, say $i=0$, where $v\left(g_{0}\right)=1, \nu_{0}=1$.

Let $h$ be any element in $F$ and let $\mu_{i}$ be the order of $h$ at $p_{i}$. Then $h /\left[\prod g_{i}^{\mu_{i}}\right]$ is neither zero nor infinite at any $p_{i}$ and hence has valuation zero. Thus $v(h)=\mu_{0}$ the order of $h$ at $p_{0}$, proving the proposition.

Proposition 2. Let $F$ be the field of quotients of the bounded analytic functions on a noncompact finite Riemann surface $W$. Then to each valuation $v$ on $F$ there is a unique point $p_{0} \in W$ such that $v(f)$ is the order of $f$ at $p_{0}$.

Proof. If $f$ is an analytic function such that $|f|<M$ on $W$, then for each $\lambda$ with $|\lambda| \geqq M$, the function $f-\lambda$ has an $n$th root in $F$ for each positive integer $n$. Thus $v(f-\lambda)$ is divisible by every $n$ and so must be zero. By (ii) we have $v(f) \geqq 0$, showing that $v$ is non-negative on the bounded functions.

Let $f$ be the function of Lemma 2. Then by (iii) there is a complex number $z_{0}$ such that $v\left(f-z_{0}\right)>0$. Since $v(f-\lambda)=0$ whenever $|\lambda| \geqq 1$, we have $\left|z_{0}\right|<1$. Let $p_{0}, \cdots, p_{k}$ be the points on $W$ where $f$ has the value $z_{0}$ and let $\mu_{i}$ be the order of $f-z_{0}$ at $p_{i}$. For any bounded function $g$, the function

$$
\frac{\Pi\left(g-g\left(p_{i}\right)\right)^{\mu_{i}}}{f-z_{0}}
$$

is bounded, and so $v\left(g-g\left(p_{i}\right)\right)$ must be positive for some $i$. Thus if $g$ is a bounded function and $v(g)>0$, then $g$ vanishes at one of the points $p_{i}$.

Let $g_{0}$ be a bounded function which separates the $p_{i}$. By subtracting 
a suitable constant we may assume $v\left(g_{0}\right)>0$. Then $g_{0}$ must have a zero at precisely one of the $p_{i}$, say $p_{0}$. If $g$ is any bounded function such that $g\left(p_{0}\right) \neq 0$, then for a sufficiently small $\epsilon$ the function $g_{0}+\epsilon g$ vanishes at none of the points $p_{i}$. Thus

$$
0=v\left(g_{0}+\epsilon g\right) \geqq \min \left[v\left(g_{0}\right), v(g)\right],
$$

whence $v(g)=0$. Consequently, $v(g)>0$ implies that $g\left(p_{0}\right)=0$. If on the other hand $g\left(p_{0}\right)=0$, we may subtract a constant $\lambda$ so that $v(g-\lambda)>0$. But then $g-\lambda$ must vanish at $p_{0}$, whence $\lambda=0$ and $v(g)>0$. Thus for bounded functions $v(g)>0$ if and only if $g\left(p_{0}\right)=0$.

Now let $g$ be an analytic function on $\bar{W}$ with a simple zero at $p_{0}$ and no other zeros on $\bar{W}$. If $h$ is a bounded analytic function of order $\nu$ at $p_{0}$, then $h /\left(g^{\nu}\right)$ is a bounded analytic function which does not vanish at $p_{0}$. Hence its valuation is zero, and $v(h)=v v(g)$. Since each function in $F$ is the quotient of two bounded functions we see that its valuation is just its order at $p_{0}$ times $v(g)$. Since $v$ is onto, $v(g)=1$, and the proposition is proved.

4. Homomorphisms between rings of meromorphic functions. In this section we discuss some of the consequences of homomorphisms between rings of meromorphic functions on two Riemann surfaces. We first prove a proposition which tells us that in general a ring homomorphism must be an algebraic (or conjugate algebraic) homomorphism.

Proposition 3. Let $R_{1}$ and $R_{2}$ be any two rings of meromorphic functions on Riemann surfaces $W_{1}$ and $W_{2}$, and let $\phi$ be a ring homomorphism of $R_{1}$ into $R_{2}$. If $R_{1}$ contains all the complex constants, then $\phi$ takes constant functions into (possibly different) constant functions. If in addition $R_{1}$ contains a bounded function $f$ such that $\phi(f)$ is not constant and if $R_{1}$ contains all functions of the form $F \circ f$, where $F$ is analytic on the range of $f$, then either $\phi(\lambda)=\lambda$ for each complex constant $\lambda$ or else $\phi(\lambda)=\bar{\lambda}$ for each complex constant $\lambda$.

Proof. Unless the range of $\phi$ is 0 , we must have $\phi(1)=1$. From this it follows that $\phi(\rho)=\rho$ for each real rational $\rho$ and that $\phi(i)$ is either $i$ or $-i$. For each rational $\rho$, i.e. a number of the form $\alpha+i \beta, \alpha$ and $\beta$ real rational, we have $\phi(\rho)=\rho$ in the first case and $\phi(\rho)=\bar{\rho}$ in the second. If $\lambda$ is any complex constant, then $\lambda-\rho$ has a square root for any rational $\rho$, and thus for each rational $\rho$ the function $\phi(\lambda)-\rho$ has a square root. Consequently $\phi(\lambda)$ must be constant.

Let $f$ be the bounded analytic function given in the second part of the proposition. Without loss of generality we may assume that 0 is in the range of $\phi(f)$. Since $\phi(f)$ is not constant there must be a positive 
$\delta$ such that each number whose modulus is less than $\delta$ is in the range of $\phi(f)$. Let $M$ be the supremum of $|f|$, and for any complex constant $\lambda$ take a real rational $\rho$ such that $\rho M|\lambda|<1$. Then the function $g=1+\rho \lambda f$ has an $n$th root for every $n$. Consequently the function $\phi(g)=1+\rho \phi(\lambda) \phi(f)$ must also have an $n$th root for every $n$. But this implies that $[\rho \phi(\lambda)]^{-1}$ is not in the range of $\phi(f)$, and so $\rho|\phi(\lambda)|$ must be less than $1 / \delta$, whence

$$
|\phi(\lambda)|<1 / \delta \rho .
$$

Since this is true for all positive rational $\rho$ which are less than $(M|\lambda|)^{-1}$, we have

$$
|\phi(\lambda)| \leqq M|\lambda| / \delta
$$

Thus $\phi$ is a continuous homomorphism of the complex field onto itself, and so we must have $\phi(\lambda)=\lambda$ for all $\lambda$ or else $\phi(\lambda)=\bar{\lambda}$ for all $\lambda$.

Proposition 4. Let $W_{1}$ be a Riemann surface and $F_{1}$ a field of meromorphic functions on it with the property that for each valuatiou $v$ on $F_{1}$ there is a unique point $p \in W_{1}$ such that $v(f)$ is the order of $f$ at $p$. Then for any algebraic homomorphism ${ }^{3} \phi$ of $F_{1}$ into the field $F_{2}$ of all meromorphic functions on a Riemann surface $W_{2}$, there is an analytic mapping $\psi$ of $W_{2}$ into $W_{1}$ such that $\phi f=f \circ \psi$.

Proof. Since $\phi$ is one-to-one, the range of $\phi$ contains nonconstant functions. Consequently, for each $p \in W_{2}$ the function which gives the order of $\phi(f)$ at $p$ is a mapping of $F_{1}$ into the integers which is either a valuation on $F_{1}$ or a positive integer times a valuation. By hypothesis there is a point $\psi(p) \in W_{1}$ such that this valuation is just the order of a function at $\psi(p)$. Thus $\phi(f)$ vanishes at $p$ if and only if $f$ vanishes at $\psi(p)$. Consequently $\phi f=f \circ \psi$. The mapping $\psi$ is readily seen to be analytic, proving the proposition.

Corollary. Let $W_{0}$ be a compact Riemann surface and $F_{0}$ its field of meromorphic functions. Then for any algebraic homomorphism $\phi$ of $F_{0}$ into the field $F_{2}$ of all meromorphic functions on a Riemann surface $W_{2}$ there is an analytic mapping $\psi$ of $W_{2}$ into $W_{0}$ such that $\phi(f)=f \circ \psi$.

Theorem 1. Let $W_{0}$ be a compact Riemann surface, and $W_{1}$ a connected open subset of $W_{0}$ with the property that for each boundary point of $W_{1}$ there is a bounded analytic function on $W_{1}$ having an essential singularity at that point. Let $F_{1}$ be the field of quotients of the bounded

\footnotetext{
${ }^{3}$ i.e. a homomorphism $\phi$ with the property that $\phi(\lambda)=\lambda$ for each complex constant $\lambda$.
} 
analytic functions on $W_{1}$, and let $F_{2}$ be the field of all meromorphic functions on some Riemann surface $W_{2}$. Let $\phi$ be a ring homomorphism of $F_{1}$ into $F_{2}$ such that $\phi(i)=i$. Then there is an analytic mapping $\psi$ of $W_{2}$ into $W_{1}$ such that $\phi(f)=f \circ \psi$.

Proof. By Proposition 3, $\phi(\lambda)=\lambda$ for each complex constant $\lambda$, and we have an algebraic homomorphism.

We first note that the field $F_{0}$ of meromorphic functions on $W_{0}$ is contained in $F_{1}$. For let $p$ be a fixed point of $W_{1}$. Then each function in $F_{0}$ is a rational function of functions in $F_{0}$ which have poles only at $p$. But if $g \in F_{0}$ has a pole of order $n$ at $p$ and no other poles, and if $f$ is a bounded analytic function on $W_{1}$, then $[f-f(p)]^{n} g$ is also a bounded analytic function on $W_{1}$, whence $g$ is a quotient of two bounded analytic functions on $W_{1}$.

Thus $\phi$ maps $F_{0}$ into $F_{2}$, and by the corollary of Proposition 4 there is an analytic mapping $\psi$ of $W_{2}$ into $W_{0}$ such that $\phi(f)=f \circ \psi$ for $f \in F_{0}$.

We next note that $\phi$ carries bounded functions into bounded functions. For if $f \in F_{1}$ is bounded by $M$, then $f-\lambda$ has an $n$th root whenever $|\lambda|>M$. But this implies $\phi(f)-\lambda$ has an $n$th root whenever $|\lambda|>M$, whence $|\phi(f)| \leqq M$ on $W_{2}$.

Let $p \notin \bar{W}_{1}$, and let $g$ be a function in $F_{0}$ which has a pole only at $p$. Then $f$ is bounded on $W_{1}$, whence $f$ must be bounded on $W_{2}$. This implies $p \notin \psi\left(W_{2}\right)$, and so $\psi\left(W_{2}\right) \subset \bar{W}_{1}$.

Let $f$ be a bounded function in $F_{1}$. Since $\psi$ is an open mapping and $\psi\left(W_{2}\right) \subset \bar{W}_{1}$, we can find a point $p \in W_{2}$ such that $\psi(p) \in W_{1}$. Let $g$ be a function in $F_{0}$ which has no poles except at $\psi(p)$ where it has a pole of order $n$. Then $\{f-f[\psi(p)]\}^{n} g$ is a bounded function and so its image under $\phi$ must also be bounded. But this image is $\{\phi(f)-f[\psi(p)]\}^{n} \phi(g)$, and $\phi(g)$ has a pole at $p$. Hence at $p$ we must have $\phi(f)=f[\psi(p)]$.

Thus $\phi(f)=f \circ \psi$ in $\psi^{-1}\left(W_{1}\right)$. If $p$ is a boundary point of $W_{1}$, we let $f$ be a function in $F_{1}$ which has an essential ${ }^{4}$ singularity at $p$. Then if $p$ were the image under $\psi$ of some point $q \in W_{2}$, the function $\phi(f)$ would have to have an essential singularity at $q$, contrary to the fact that $\phi(f)$ is in $F_{2}$ and therefore meromorphic at $q$. This implies that $\psi\left(W_{2}\right) \subset W_{1}$, and the proof is complete.

4 The term "essential singularity" here excludes algebraic branch points as well as poles. However, if to each boundary point of $W_{1}$ there is a bounded analytic function in $W_{1}$ with a (possibly algebraic) singularity at that point, then the method of condensation of singularities enables us to construct a bounded analytic function in $W_{1}$ which has an essential singularity at each boundary point of $W_{1}$. 


\section{BiBLIOGRAPHY}

1. M. Heins, Algebraic structure and conformal mapping, Technical Report issued by the Institute for Advanced Study, 1957.

2. H. L. Royden, Rings of analytic and meromorphic functions, Trans. Amer. Math. Soc. vol. 83 (1956) pp. 269-276.

3. W. Rudin, Some theorems on bounded analytic functions, Trans. Amer. Math. Soc. vol. 78 (1955) pp. 333-342.

4. H. Weyl, Die Idee der Riemannschen Flaechen, Berlin, 1923.

STANFORD UNIVERSITY

\section{A SEMI-SIMPLE MATRIX GROUP IS OF TYPE $I$}

\section{W. FORREST STINESPRING}

The purpose of the following note is to give a short simple proof of the most important special case of Harish-Chandra's result [3, Theorem 7] that every connected semi-simple Lie group is of type $I$. We shall prove that every continuous unitary representation of a connected semi-simple matrix group is of type $I$. This fact is a consequence of theorems in two papers of Godement [1, Theorem 2; 2 , Theorem 8]. An improved version of Godement's method is used here. The improvements are:

(1) the argument is considerably shorter;

(2) infinite dimensional nonunitary representations are not needed;

(3) the argument is completely global; no direct integrals are used. These improvements seem not to have been noticed before.

Definition. We shall say that an algebra is of type $I_{s n}$ if it satisfies the identities

$$
\left[A_{1}, \cdots, A_{r}\right]=\sum_{i_{1}, \cdots, i_{r}} \operatorname{sgn}\left(\begin{array}{c}
1 \cdots r \\
i_{1} \cdots i_{r}
\end{array}\right) A_{i_{1}} A_{i_{2}} \cdots A_{i_{r}}=0
$$

for all $r$ for which the algebra of all $n \times n$ matrices satisfies them.

In [5, §2], Kaplansky shows that the algebra of all $n \times n$ matrices is of type $I_{\leqq n}$ but not of type $I_{\leqq n-1}$. It follows from the definition that an algebra is of type $I_{\Xi n}$ if it is a subalgebra of an algebra of type $I_{\leqq n}$, or if it is a homomorphic image of an algebra of type $I_{\unlhd n}$, or if it has a separating family of homomorphisms into algebras of type $I_{\leqq n}$. Since the above identities are linear in each variable, a von Neumann algebra is of type $I_{\leqq n}$ if it has a weakly dense subalgebra of type $I_{\leqq n}$. A von Neumann algebra of type $I$ (in the usual sense) is

Received by the editors May 5, 1958. 